KOMPASS

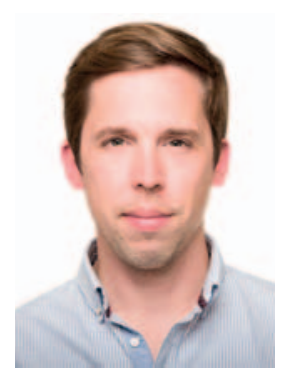

Dr. med. Matthias Raspe,

Koordination «PneumoCampus»

\title{
Scharfe Blicke, kluge Initiativen, klare Ansagen: Nur Mut!
}

Herzlich willkommen zur dritten Ausgabe des «PneumoCampus» 2017, in der wir Ihnen unsere neue Reihe «Blickdiagnosen» vorstellen wollen. In jeder Ausgabe des «PneumoCampus» wird ein Befund mit einer kurzen Beschreibung präsentiert. Sie sind dabei gefragt, die richtige Diagnose zu stellen. Die Auflösung schließt gleichermaßen den Rahmen nach dem letzten Campus-Beitrag. Wir wünschen viel Freude und Erfolg dabei, unsere spannenden Fälle zu lösen! Mit fortgeschrittener Behandlungspraxis dürfte Ihre Quote richtiger Antworten steigen. Doch eine gute Weiterbildungsqualität umfasst weit mehr als die Summe richtig beantworteter Fragen; sie soll schließlich und vor allem zu einer qualitativ hochwertigen Versorgung unserer Patienten führen. Prof. Michael Denkinger, Chefarzt der Geriatrie und Ärztlicher Direktor der Agaplesion Bethesda Klinik in Ulm, hat mit Kollegen ein Mastertrainerkonzept entwickelt, dass die Weiterbildung in den Kliniken vor Ort vor allem durch mehr Struktur verbessern soll. Die Hintergründe und das Konzept erläutert er im Interview.

Auch der bvmd (Bundesverband der Medizinstudierenden in Deutschland) sieht die derzeitige wie geplante Infrastruktur für eine fundierte Ausbildung kritisch und formuliert für «PneumoCampus» eine Stellungnahme zum durchaus kontrovers diskutierten Masterplan Medizinstudium 2020, der das Medizinstudium fit für die Zukunft machen soll. Insbesondere Maßnahmen, die den Nachwuchs in der Allgemeinmedizin fördern sollen, bieten Potenzial für intensive Diskussionen.

Die Nachwuchsgruppe der Deutschen Gesellschaft für Innere Medizin hat sich beim 123. DGIMKongress 2017 in Mannheim ebenfalls Gedanken über Rahmenbedingungen ärztlicher Ausbildung gemacht und stellt in diesem Heft einen kurzen Abriss der diskutierten Themen vor. Am anderen Spektrum der medizinischen Karriere stehen die Kriterien guter wissenschaftlicher Arbeit: Wer regelmäßig publiziert, wird für gewöhnlich im Verlauf durch das Journal gebeten, selbst als Gutachter für eingereichte Manuskripte tätig zu werden. Dieses Geben und Nehmen ist die tragende Säule des Peer-Review-Verfahrens. In diesem dritten und letzten Beitrag unserer Serie zum wissenschaftlichen Publizieren erörtert Dr. Sven Riestenpatt, Cheflektor im Karger Verlag, wesentliche Informationen zum Ablauf und Verfassen von Manuskript-Gutachten.

Ich wünsche Ihnen eine anregende Lektüre! Mit besten Grüßen,

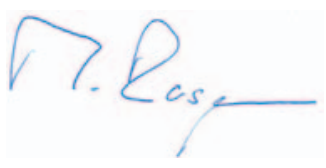

\section{KARGER}

Fax +497614520714 information@karger.com www.karger.com (c) 2017 S. Karger GmbH, Freiburg
Herrn Dr. med. Matthias Raspe

Medizinische Klinik

Schwerpunkt Infektiologie und Pneumologie

Charité - Universitätsmedizin Berlin

Augustenburger Platz 1, 13353 Berlin, Deutschland matthias.raspe@charite.de 


\section{Blickdiagnosen}

\section{Welche Diagnose vermuten Sie?}

Eine 23-jährige Frau stellt sich mit den führenden Symptomen Husten/Kratzen im Hals seit 4-6 Monaten und einer zunehmenden Luftnot unter Belastung seit etwa 2 Monaten in der Notaufnahme vor. Es bestehen keine relevanten Vorerkrankungen. Als einzige Medikation wird ein Kontrazeptivum eingenommen. Die Patientin ist Nieraucherin. Die körperliche Untersuchung ergibt keinen wegweisenden Befund. Bei erhöhten D-Dimeren (ansonsten im Labor auffällig: CRP mit 30mg/l, Ref.: <5) wird über die Notaufnahme eine Computertomographie des Thorax mit Kontrastmittel veranlasst. Die Röntgen-Thorax-Aufnahme stammt aus dem gleichen Zeitraum.

\section{Auflösung:}

Im Anschluss an den letzten Beitrag des «PneumoCampus» (print) bzw. unter diesem Beitrag zum Anklicken (online).

Kontaktadresse: Herrn Dr. med. Matthias Raspe, Medizinische Klinik, Schwerpunkt Infektiologie und Pneumologie, Charité - Universitätsmedizin Berlin, Augustenburger Platz 1, 13353 Berlin, Deutschland, matthias.raspe@charite.de

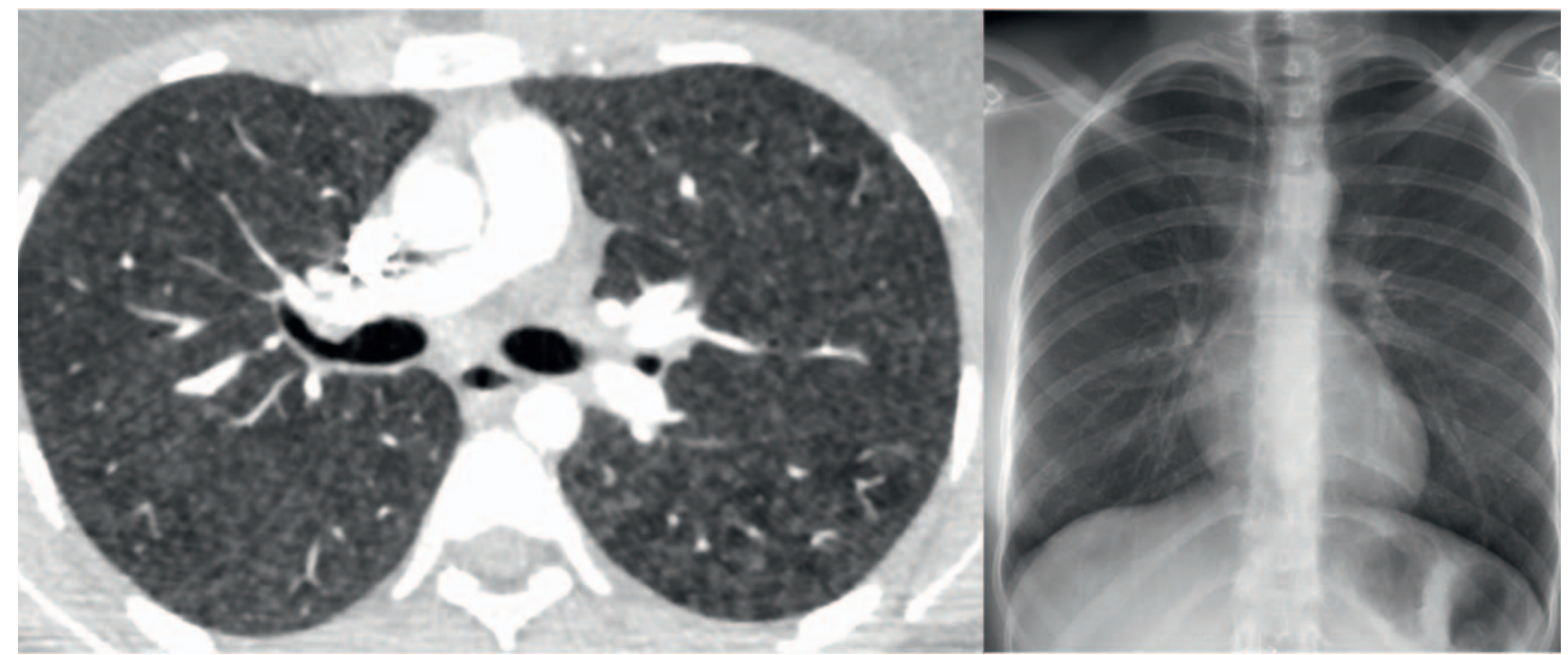

Bildbefunde mit freundlicher Genehmigung von Dr. med. C. Althoff, Leitung Interventionsradiologie Charité Campus Mitte, Institut und Klinik für Radiologie CCM und CVK, Charité - Universitätsmedizin Berlin

\section{Interview mit Prof. Dr. Michael Denkinger \\ Das Mastertrainer-Konzept für eine transparente und realitätsnahe ärztliche Ausbildung}

Je besser die Weiterbildung, desto besser der Arzt - so weit, so verständlich. In Deutschland wird die Weiterbildung junger Ärzte durch die Weiterbildungsordnung qualitativ gesichert. Doch ergaben zahlreiche Umfragen in den letzten Jahren, dass diese Weiterbildungsordnung verschiedenste Schwachstellen aufweist, wie z.B. eine mangelnde Arbeit mit Logbüchern oder unklare Rotationspläne.

Um die Qualität der Weiterbildung direkt an den Kliniken zu verbessern, wurde 2013 von Prof. Dr. Marcus Siebold, Dr. med. Jörg Ansorg und Prof. Dr. Michael Denkinger das Mastertrainerprogramm initiiert. Kern des Konzepts ist es, den Weiterbildungsbefugten das Know-how zu vermitteln, wie man gute Weiterbildung strukturieren und durchführen kann. Gleichzeitig werden sie selbst zu Mastertrainern ausgebildet, die das Programm ihrerseits an ihren Kliniken oder in eigenen Kursen weitervermitteln können. Bisher sind in den zweitätigen Kursen ungefähr 90 Mastertrainer ausgebildet worden. Zweimal im Jahr finden zusätzlich Su- pervisionen statt, die den ausgebildeten Mastertrainern dabei helfen, das Gelernte anzuwenden und zu konsolidieren. Bisher beteiligen sich der Berufsverband der Deutschen Internisten, der Berufsverband der Deutschen Chirurgen, der Berufsverband der Unfallchirurgen und der Berufsverband der Chirurgen Österreichs an dem Projekt. Um es auch anderen Berufsverbänden zu ermöglichen, dieses Konzept zu nutzen, befindet sich aktuell das «Bündnis für Qualität in der Facharztweiterbildung» in der Gründungsphase.

Was sind die Schwachstellen der ärztlichen Weiterbildung? Was ist das größte Problem?

Zuerst einmal muss man sagen: Es ist nicht alles schlecht. Wir haben in Deutschland ein sehr offenes System, das - zumindest theoretisch - auch klar kriteriengeleitet ist. Allerdings haben wir ein Problem mit der Umsetzung und mit der Steuerung der Umsetzung 
vor Ort. Ein weiteres Problem ist die Überprüfung der vermittelten Inhalte: Wir prüfen dies anhand von Zahlen und absolvierten Zeiten über Logbücher und Zeugnisse, die von den Weiterbildungsermächtigten ausgefüllt werden. Umfragen zeigen aber, dass die Logbücher nicht immer gut geführt sind und dass es außerdem Probleme damit gibt, auf die geforderten Mindestzahlen zu kommen. Zudem fehlt eine Leistungsreflexion, wo regelmäßig systematisch hinterfragt wird, was gut und was schlecht läuft. An diesen Punkten setzen wir mit dem Mastertrainerprojekt an.

\section{Kann die Novellierung der Musterweiterbildungsordnung einen grundsätzlichen Fortschritt bringen?}

Die Novellierung der Musterweiterbildungsordnung (MWBO) wird einen Fortschritt bringen, denn sie ist eher darauf ausgelegt, Kompetenzen zu vermitteln und nicht unbedingt Zeiten und Zahlen zu definieren. Wenn aber diese Kompetenzen nicht konsequent überprüft werden, wird es wieder problematisch. Dann sind Zeiten und Zahlen vielleicht doch eine bessere Überprüfung als eine nicht genau überprüfbare Kompetenz im Rahmen der 30-minütigen Facharztprüfung, an der zumindest in nächster Zeit nicht gerüttelt werden soll. Und bei 6 Jahren Weiterbildung ist es natürlich nicht möglich, den Kandidaten in diesem Zeitrahmen auf Herz und Nieren zu prüfen. Wir wollen alle gut qualifizierte Fachärzte, überprüfen das aber auch nach der Einführung der neuen MWBO lediglich über die Logbücher und die Facharztprüfung. So wird es im Zweifel sogar noch schwieriger werden, darzustellen, was die Leute in den Häusern wirklich geleistet haben.

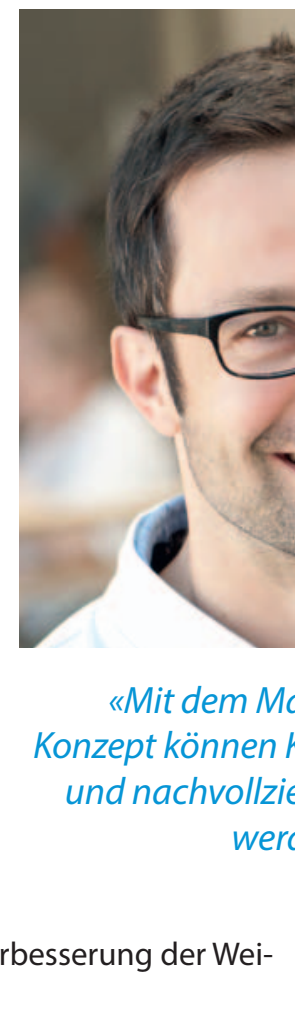

Wo oder wie kann man
terbildung erreichen?

Ich glaube natürlich, dass das am ehesten mit der Struktur aus unserem Projekt geht. Das Projekt besteht im Grunde aus 4 wesentlichen Teilen. Der erste ist das Kerncurriculum. Hier wird klar definiert, was dem Assistenten zu welchem Zeitpunkt der Weiterbildung beigebracht werden soll. Damit wird die Weiterbildung nicht nur zeitlich klar strukturiert, sondern auch inhaltlich. Man kann so auch abschätzen, welche Dinge nicht innerhalb der Klinik zu schaffen sind. Wenn z.B. klar wird, dass der Weiterbildungsassistent nicht genügend Sonographien in seiner Klinik machen kann, kann man über andere Strategien nachdenken, beispielsweise eine Hospitation oder einen Kurs. Mit dem Kerncurriculum ist es dann auch nicht so dramatisch, wenn die Gesamtzahl der gemachten Sonographien nicht ganz der geforderten Anzahl entspricht. Was der Assistent nachweislich leisten kann, wird ihm als Kompetenz im Kerncurriculum und im Logbuch bescheinigt. Zusätzlich kann man durch das Kerncurriculum auch die Weiterbildung mit dem QM-System verknüpfen. Bisher läuft beides parallel, was keinen Sinn ergibt, denn
Arbeits- und Verfahrensanweisungen gehören zu dem, was ein Arzt kennen und können muss. Daran schließt der zweite wichtige Teil des Mastertrainerkonzepts an: die Soft Skills, die ein Arzt haben muss, wie Empathie, Verständnis des Gesundheitssystems, Teamfähigkeit usw. Diese personellen ärztlichen Schlüsselkompetenzen orientieren sich an den ACGME-Kriterien (Accrediation Council for Graduate Medical Education) und sind ebenfalls Teil des Kerncurriculums.

An die Überprüfung der Kompetenzen schließt der nächste wesentliche Teil an: die Lernstandsrückmeldung; sie gehört integral zu den jährlichen Weiterbildungsgesprächen, die es schon gibt. Entscheidender Punkt im Mastertrainersystem ist jedoch, dass diese Rückmeldung klar kriteriengeleitet ist. Hier werden die Punkte aus dem ACGME-Kriterien und dem Kerncurriculum zusammengeführt und anhand einer 5-Punkte-Skala abgefragt. Hinzu kommt, dass bei diesen Rückmeldungen nicht nur die Fremdeinschätzung des Weiterbilders gefragt ist, sondern auch die Eigeneinschätzung des Assistenten. Im Vergleich der beiden Einschätzungen kann man dann gut sehen, wo es Differenzen gibt und wo der Assistent vielleicht noch einmal nachfassen muss - aber auch wo in der Weiterbildung selbst vielleicht etwas geändert oder ergänzt werden sollte.

Wer möchte, kann bei verschiedenen, gut operationalisierbaren Punkten, wie etwa der Sonographie, operativen Eingriffen etc. diese aktiv in Testaten überprüfen, die den vierten Hauptpunkt des Mastertrainersystems bilden. Die Prüfung kann innerhalb von wenigen Minuten während einer regulär anstehenden Untersuchung erfolgen, sollte sich aber auf wenige Punkte im Kerncurriculum beschränken und angekündigt werden. Für solche Testate gibt es dann auch Kriterienraster, anhand derer die Leistung klar bewertet werden kann und womit auch klar nachvollzogen werden kann, was der Assistent gut gemacht hat oder an welchen Stellen er noch etwas verbessern muss - ohne pauschales «gut» oder «schlecht». Genau das ist die Besonderheit des Mastertrainersystems: Es strukturiert die Weiterbildung und liefert eine Kriterienliste, mit der Kompetenzen klar und nachvollziehbar überprüft werden können. An der gesamten Weiterbildung selbst ändert es maximal 20\%, da das meiste auf bereits Vorhandenem aufbaut.

Die Hauptschwierigkeit dieses Systems liegt allerdings in der Umsetzung und der Nachhaltigkeit. Es ist Projektmanagement gefragt, und man muss Zeit investieren - für die Lernückstandsmeldungen, die Testate usw. Aber ich glaube, das ist dennoch ein überschaubarer Aufwand und letztlich gute Zeitinvestition.

\section{Wie sehen die nächsten Schritte der Initiative aus?}

Der nächste Schritt ist, dass die Mastertrainer selbst anfangen, solche Veranstaltungen anzubieten und ihrerseits das Ganze weitertragen. Prof. Siebolds (Gesundheitsökonom, Katholische Hoch- 
schule NRW in Köln) hat dafür eine Art Konzeptpapier mit Beispieldramaturgien zusammengestellt, sodass die Trainer eine Schulung auch selbst veranstalten können. Es gibt unter den Mastertrainern einige Motivierte, die das machen möchten, das sind natürlich die, die das System auch in ihren Kliniken entsprechend umgesetzt haben. Ein paar andere tun sich da schwerer. Um dieses «Schneeballsystem» zu erreichen, sind wir auch dabei, das «Bündnis für Qualität in der Facharztweiterbildung» zu gründen, um eine Plattform zu bieten und «dranbleiben» zu können.

Wäre es sinnvoll, wenn das Programm irgendwann auch «offiziell» in die Weiterbildungsordnung eingegliedert wird?

Ja, die Ärztekammern haben auch Interesse bekundet. Beispielsweise könnte man jedem, der eine Weiterbildungsermächtigung beantragt, einen Tageskurs zu unserem Mastertrainersystem an die
Hand geben, wofür wir aber mehr Mastertrainer benötigen würden. Möglich wäre auch, dass die Kammern das selbst mitgestalten. Wenn sie das zusammen mit den Berufsverbänden übernehmen würden, würde das Projekt sicherlich an Fahrt gewinnen. Aber auch das Einbinden weiterer Berufsverbände in unser neues Bündnis würde helfen - Sie sind herzlich eingeladen!

\section{Vielen Dank für das Interview!}

Kontaktadresse: Prof. Dr. med. Michael Denkinger, Mitglied des Vorstandes im Berufsverband Deutscher Internisten e.V., Schöne Aussicht 5, 65193 Wiesbaden und AGAPLESION Bethesda Klinik, Geriatrie der Universität Ulm, Geriatrisches Zentrum Ulm/Alb-Donau, Zollernring 26, 89073 Ulm, Deutschland, info@bdi.de (Stichwort «Mastertrainer Weiterbildung») 
Die bvmd begrüßt das gemeinsame Ziel von Bund und Ländern, das Medizinstudium moderner und innovativer zu gestalten und eine kompetenzorientierte und qualitativ hochwertige Ausbildung zu ermöglichen. Wir Studierende haben den mehr als zwei Jahre andauernden, internen Verhandlungen die offizielle Verkündung des Masterplans am 31. März 2017 durch Bundesgesundheitsminister Hermann Gröhe, Bundesforschungsministerin Prof. Johanna Wanka sowie Vertreterinnen und Vertreter der Gesundheits- und der Kultusministerkonferenz mit großer Spannung erwartet. Die mit dem Eckpunktepapier geplante Reform ist eine Chance, sich der Neuordnung des Zulassungsverfahrens, der praxisnahen Ausrichtung der medizinischen Ausbildung und der longitudinalen Verankerung der Allgemeinmedizin im Studium zu widmen.

Wir Studierende sind erfreut, dass Praxis und Theorie bereits von Beginn des Studiums an miteinander verbunden werden sollen. Auch die Ausrichtung der Lehrinhalte auf ein Kerncurriculum, die Implementierung des Nationalen Kompetenzbasierten Lernzielkatalogs Medizin (NKLM) sowie die Förderung der Wissenschaftlichkeit im Studium sind seit Langem von der bvmd geforderte wichtige und richtige Reformen. Damit die große Reform des Medizinstudiums eine Verbesserung des Studiums bewirkt, bedarf es langfristig der Bereitschaft der Länder und des Bundes, die nötige Finanzierung für die Fakultäten zu sichern. Die bvmd bedauert, dass mit dem Masterplan Medizinstudium 2020 die Gelegenheit für eine Etablierung einer fairen Regelung von Fehlzeiten und flächendeckend einheitlichen Aufwandsentschädigungen im Praktischen Jahr vertan wird. Zudem befürchten wir, dass durch die Diskussion um einzelne symbolpolitische Maßnahmen, wie die Einführung einer Landarztquote und die Pflichtprüfung Allgemeinmedizin im dritten Staatsexamen (M3), der Gedanke einer umfänglichen Reform des Medizinstudiums in den Hintergrund geraten könnte. Die bvmd ist davon überzeugt, dass mit einer Landarztquote lediglich die Gründe für den Landarztmangel kaschiert würden. Statt einer Quote braucht es eine Steigerung der Attraktivität von Arbeits- und Lebensbedingungen in unterversorgten und von Unterversorgung bedrohten Regionen, um Versorgungsengpässen gezielt entgegenzuwirken. Bei der Berufswahl angehender Ärztinnen und Ärzte spielen neben der Attraktivität der Arbeitszeiten und Weiterbildungsmöglichkeiten auch die Vereinbarkeit von Beruf und Familie, das wissenschaftliche Umfeld, eine kollegiale Atmosphäre, ein kooperatives und nicht zu hierarchisches Betriebsklima und die Minimierung nichtärztlicher Verwaltungstätigkeiten eine essenzielle Rolle.

Wir Studierende wünschen uns von den Verantwortlichen von Bund und Ländern, dass bei der Umsetzung der Maßnahmenvorschläge in die ärztliche Approbationsordnung die Ideen der Medizinstudierenden Gehör finden und dass auf die Veröffentlichung der 37 Maßnahmen des Masterplans Medizinstudium 2020 sowohl ein umfassender als auch ein zeitnaher Umsetzungs- und Finanzierungsplan folgt.

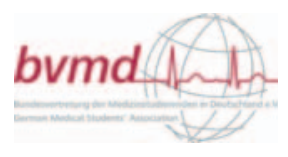

Kontaktadresse: Carolin Siech, Bundesvertretung der Medizinstudierenden in Deutschland e.V. (bvmd), Robert-Koch-Platz 7, 10115 Berlin, Deutschland, pr@bvmd.de.

Programm für den internistischen Nachwuchs

\section{Der 3. Tag der Jungen Internisten beim 123. DGIM-Kongress 2017 in Mannheim}

Beim diesjährigen 123. Hauptkongress der Deutschen Gesellschaft für Innere Medizin (DGIM) Ende April 2017 in Mannheim hatte deren Nachwuchsgruppe zum dritten Mal Gelegenheit, während des Kongresses ein eigenes Programm von jungen und für junge Internisten umzusetzen.

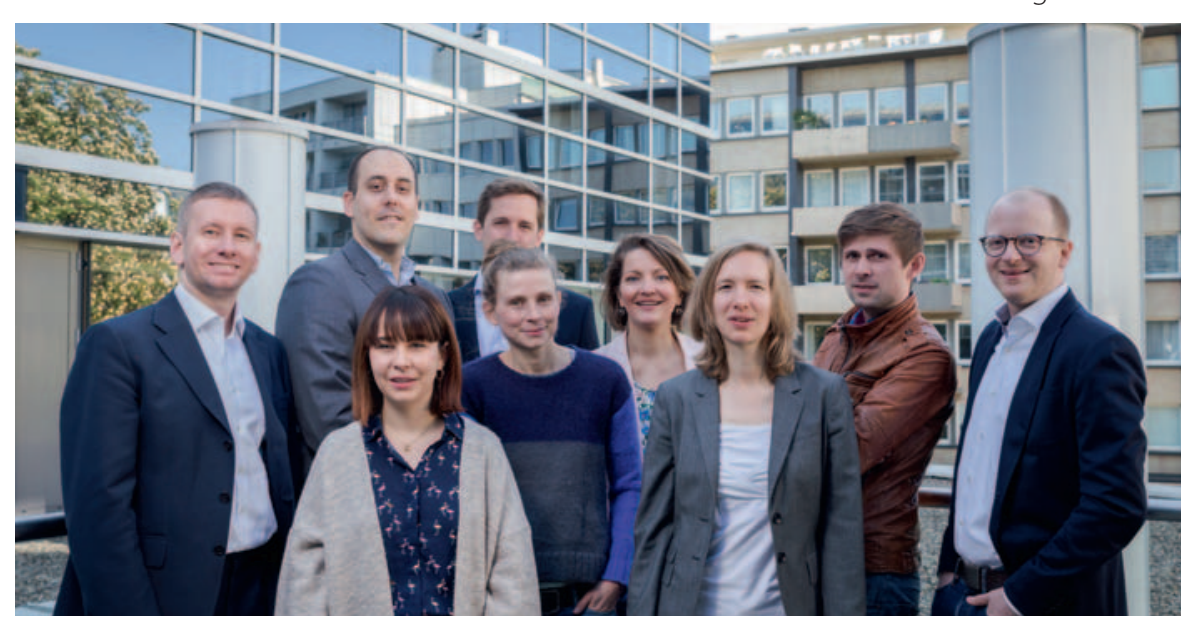

Das Programm bestand in diesem Jahr aus 3 übergeordneten Themenblöcken mit 90 min Dauer am Kongresssamstag und einem Extra-Block am Folgetag. Der Tag der Jungen Internisten wurde wieder als Teil des etablierten «Chances - Forum für junge Mediziner» ausgerichtet.

Im ersten Themenblock, dem sogenannten klinischen Gymnasium, drehte sich alles um konkrete und interaktive Fallschilderungen aus der Klinik. In weiteren Vorträgen wurden die wichtigsten nephrologischen Krankheitsbilder und das Zentrum für seltene und unbekannte Erkrankungen in Marburg vorgestellt.

Im zweiten Themenblock mit dem Titel «Spannungsfeld Familie und Beruf» wurden Daten einer kürzlich durchgeführten eigenen Befragung unter 1600 Ärzten zu diesem Thema präsentiert. Außerdem teilten eine Assistenzärztin und eine Chefärztin ei- 
nen persönlichen Erfahrungsbericht zu diesem Thema. Vor der abschließenden Podiumsdiskussion wurde weitere wichtige Studienergebnisse zum Thema vorgestellt.

Im dritten Block ging es um Methoden in der Pneumologie anhand zweier exzellenter Vorträge über die nicht-invasive Beatmung und die Thoraxsonographie.

Im Extra-Block am Folgetag mit dem Titel «Sonographie für junge Internisten» wurden die wichtigsten Befunde aus der Gefäßdiagnostik, der Abdomensonographie und der transthorakalen Echokardiographie für junge Internisten präsentiert.

Auch in diesem Jahr fand die Veranstaltung regen Anklang - und das nicht nur bei den Kolleginnen und Kollegen in Weiterbildung - und war durchgehend gut besucht. Wir freuen uns im kommenden Jahr mit dem 4. Tag der Jungen Internisten der DGIM das Konzept eines vielseitigen Programms nach den Bedürfnissen junger Ärztinnen und Ärzte in internistischer Weiterbildung fortzusetzen!

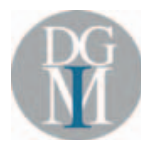

Kontaktadresse: Herrn Dr. med. Matthias Raspe, Medizinische Klinik, Schwerpunkt Infektiologie und Pneumologie, Charité - Universitätsmedizin Berlin, Augustenburger Platz 1, 13353 Berlin, Deutschland, matthias.raspe@charite.de

\section{S. Karger Verlag für Medizin und Naturwissenschaften GmbH Wie begutachte ich einen wissenschaftlichen Artikel?}

Wer Artikel schreibt, zur Publikation einreicht und schließlich veröffentlicht, wird unweigerlich früher oder später von den Journals, in denen er selbst veröffentlicht, gefragt werden, ob er nicht seinerseits ein eingereichtes Paper begutachten kann. Diese Gutachtereinladungen erfolgen in der Regel per E-Mail. Die Gutachten müssen dann innerhalb eines onlinebasierten Einreichungssystems eingegeben werden, das einem meist durch eigene Einreichungen schon bekannt ist.

Je nach Journal erfolgt die Begutachtung in einem einfach oder doppelt verblindeten Verfahren. Beim einfach verblindeten Verfahren hat der Gutachter Kenntnis, wer die Autoren der zu begutachtenden Studie sind, beim doppelt verblindeten Verfahren sind auch die Autoren anonymisiert. Sowohl bei dem einen wie auch bei dem anderen Vorgehen wissen aber die Autoren nicht, wer ihre Arbeit begutachtet. Selbstverständlich sind die Gutachter angehalten,

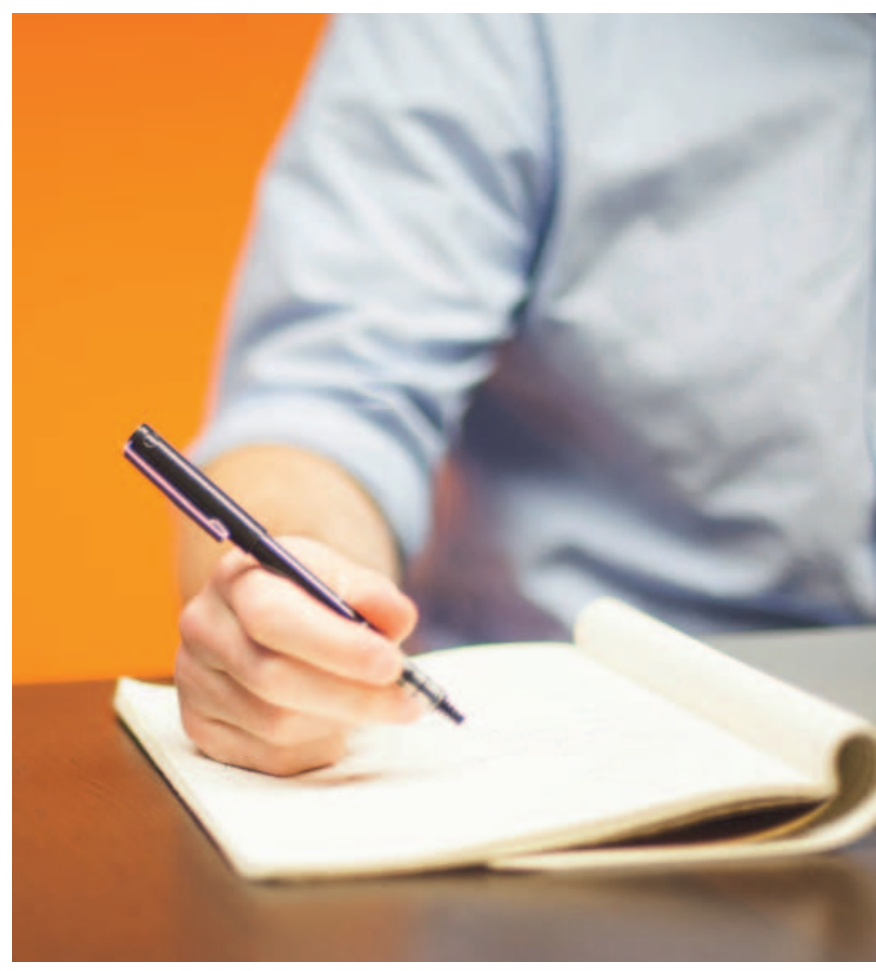

Stillschweigen in Hinblick auf die eingereichte Arbeit gegenüber jedem zu wahren, der nicht in den Begutachtungsprozess involviert ist. Im Fall von Interessenskonflikten sollte die Begutachtung abgelehnt werden. Interessenskonflikte sind z.B.:

- eine Kollaboration mit den Autoren der eingereichten Studie,

- eine direkte Konkurrenzsituation in Hinblick auf die eigene Arbeit,

- eine andauernde Animosität mit den Autoren,

- eigene finanzielle Vorteile, die mit der Publikation des zu begutachtenden Artikels verbunden sind.

Weitere Gründe für eine Ablehnung sind die Unmöglichkeit, das Gutachten innerhalb der vorgegebenen Zeit fertig zu stellen, oder eine völlige Unkenntnis der behandelten Thematik. In solchen Fällen sollten Sie dann dem anfragenden Journal mitteilen, dass Sie das Gutachten nicht übernehmen können.

Bitte bedenken Sie bei einer solchen Ablehnung, dass die grundsätzliche Bereitschaft zur Begutachtung anderer Arbeiten eine tragende Säule des Peer-Review-Verfahrens ist, zu dem es aktuell zumindest im medizinischen Bereich - keine vernünftige Alternative gibt. Durch die Absage einer Begutachtungsanfrage besteht die Gefahr, dass sich der Begutachtungsprozess einer Arbeit verlängert, und das würden Sie sich ja bei den eigenen Arbeiten auch nicht wünschen.

\section{Wie verfasse ich ein Gutachten?}

In der Regel gibt das jeweilige onlinebasierte Einreichungssystem schon eine Struktur vor. So setzt sich das Begutachtungsformular häufig aus einer Reihe von Multiple-Choice-Fragen und einem Kommentarfeld zusammen. Die Multiple-Choice-Fragen ermöglichen es dem zuständigen Associate Editor, schnell einen grundsätzlichen Eindruck von der generellen Einschätzung der Arbeit zu erhalten. Im Kommentarfeld werden Empfehlungen und Korrekturhinweise für die Überarbeitung des Artikels vermerkt, an denen sich der Autor bei der Revision seines Artikels orientieren kann. Grundsätzlich gibt es bei der Begutachtung - ebenso wie beim Schreiben eines Artikels - eine Reihe von Grundsätzen, die beachtet werden sollten. Ein hilfreiches Gutachten sollten folgende Fragen beantworten: 
- Präsentiert die Arbeit neue Befunde?

- Ist die Arbeit von Bedeutung für Forscher und Kliniker in diesem Fachgebiet?

- Ist die Arbeit von Bedeutung für Forscher und Kliniker außerhalb des Fachgebiets?

- Sind Methoden und statistische Verfahren in angemessener Weise verwendet worden?

- Wurden sämtliche ethische Standards berücksichtigt?

- Sind die Schlussfolgerungen der Autoren evidenzbasiert?

- Enthält die Arbeit falsche Informationen oder sind wichtige Dinge nicht berücksichtigt?

- Ist die aktuelle Literatur angemessen zitiert?

- Ist die Arbeit gut geschrieben, klar und leicht zu verstehen?

- Wenn die Arbeit Tabellen oder Abbildungen enthält, helfen diese dem Leser oder sind sie überflüssig?

\section{Was geschieht nach der Abgabe des Gutachtens?}

Bei einem ordentlichen Peer-Review-Verfahren gibt es zu jeder Arbeit mindestens zwei Gutachten. Liegen die Gutachten vor, wird der zuständige Associate Editor auf der Basis der Gutachten um eine abschließende Entscheidung gebeten. Weichen die Gutachten stark voneinander ab, kann er entweder selbst ein weiteres Gut- achten anfertigen oder um die Anfrage eines dritten Gutachters bitten. Im Anschluss wird der Autor über die Entscheidung informiert und erhält in diesem Zuge auch die anonymisierten Gutachten. Im Falle einer Überarbeitung wird der Autor gebeten, innerhalb eines definierten Zeitraums die revidierte Fassung seines Artikels zusammen mit einem detaillierten Antwortbrief zu den Gutachterkommentaren erneut einzureichen. Der zuständige Associate Editor prüft dann die Revision; gegebenenfalls kann er auch nochmals die Gutachter um ihre Meinung bitten. Viele Journals informieren die Gutachter nach Abschluss des Begutachtungsprozesses über die finale Entscheidung (Annahme/Ablehnung). Häufig werden den Gutachtern dabei die jeweils anderen Gutachten zu ihrer Information zur Verfügung gestellt.

Der Austausch von Informationen sowie die Koordination der Abläufe erfolgen über das Editorial Office des Verlags als neutrale schnittstelle.

Kontaktadresse: Dr. Sven Riestenpatt, Projektmanagement \& Editorial Office Transfusion Medicine and Hemotherapy / Obesity Facts, Karger Verlag GmbH S. Karger Verlag für Medizin und Naturwissenschaften GmbH, Wilhelmstraße 20a, 79098 Freiburg, Deutschland, s.riestenpatt@karger.com

\section{Auflösung Blickdiagnosen}

Im Lungenfester der Kontrastmittel-CT fallen zentri-/mikronoduläre, unscharf begrenzte Infiltrate von milchglasartiger Dichte auf. Abschnittsweise zeigt sich ein mosaikartiges Muster durch Airtrapping. Ansonsten in der CT a.e. reaktiv vergrößerte Lymphknoten bis 16 mm infracarinal und kein Nachweis einer Lungenarterienembolie. Die genaue Umfeldanamnese ergab, dass die Patientin über 4 Jahre bis eine Woche vor Diagnosestellung Wellensittiche in der Wohnung gehalten hatte. Serologisch waren die spezifischen IgG-Antikörper stark positiv. Damit lautete die Diagnose: Exogen-Allergische-Alveolitis im (CT-morphologisch) subakuten Stadium bei Sensibilisierung gegenüber Wellensittichen (sogenannte Wellensittich- bzw. Vogelhalterlunge). Das Röntgen-Thorax aus dem gleichen Zeitraum war unauffällig (laut Literatur in bis zu 30\% der Fälle beschrieben). In der initialen Lungenfunktion fielen eine restriktive Ventilationsstörung (Vitalkapazität 1,57// 50\%) und eine Verminderung des CO-Transfers auf 44\% ( $\left.T_{L C O}\right)$ auf. Neben einer strikten Antigenkarenz wurde die Therapie mit einem oralen Glukokortikoid eingeleitet. Hierunter kam es zu einer raschen Besserung der Symptomatik. 